\title{
Impacto da COVID-19 na Vida do Cardiologista e Cirurgião Cardiovascular Brasileiros
}

\author{
Impact of COVID-19 on the Life of Brazilian Cardiologists and Cardiovascular Surgeons
}

\author{
Andre Luiz Cerqueira Almeida, ${ }^{1,2}$ (๑) Marcelo Melo, ${ }^{3 \oplus}$ Rodrigo Elton Ferreira Rodrigues, ${ }^{3 \oplus}$ Luis Fábio Botelho, ${ }^{3}$ \\ Paulo André Abreu Almeida, ${ }^{4}$ Silvio Henrique Barberato ${ }^{5,6}{ }^{6}$ \\ Santa Casa de Misericórdia de Feira de Santana - Cardiologia, ${ }^{1}$ Feira de Santana, BA - Brasil \\ Sociedade Brasileira de Cardiologia Departamento de Imagem Cardiovascular, ${ }^{2}$ São Paulo, SP - Brasil \\ Universidade Federal da Paraíba, ${ }^{3}$ João Pessoa, PB - Brasil \\ UNIFACS Curso de Medicina, ${ }^{4}$ Salvador, BA - Brasil \\ Sociedade Brasileira de Cardiologia - Diretoria de Qualidade Assistencial, ${ }^{5}$ Rio de Janeiro, RJ - Brasil \\ CardioEco - Centro de Diagnóstico Cardiovascular, ${ }^{6}$ Curitiba, $P R$ - Brasil
}

\section{Introdução}

A pandemia da COVID-19 (sigla do inglês Coronavirus Disease - 2019) impactou significativamente os serviços de cardiologia. O número de consultas, exames e intervenções cardiológicas diminuiu em várias partes do mundo nos últimos meses.1,2 Contudo, apesar da pressão crescente e da carga sobre o sistema de saúde, a oferta de serviços em cardiologia não foi interrompida, já que doença cardiovascular preexistente coloca os pacientes sob maior risco de infecção, complicações e a manifestações cardíacas primárias da COVID-19. ${ }^{3}$

Além disso, os efeitos da COVID-19 têm afetado a sociedade em geral e os profissionais de saúde em particular, a saber: impacto na saúde física e mental, perturbações financeiras e alterações na qualidade de vida. ${ }^{3-5}$ Sendo assim, a pandemia causou verdadeira ruptura em diversos aspectos da prática profissional e da vida de médicos e demais profissionais de saúde. ${ }^{1,2,5}$

Nosso estudo visou avaliar o impacto causado pela pandemia de COVID-19 na vida dos médicos(as) cardiologistas e cirurgiões(ãs) cardiovasculares brasileiros(as), considerando questões ligadas à atividade profissional, renda, saúde e estilo de vida.

\section{Material e Métodos}

Os autores disponibilizaram e divulgaram um formulário online no site da Sociedade Brasileira de Cardiologia (SBC)

\section{Palavras-chave}

COVID-19; Coronavirus-19; Pandemia; Cardiologistas; Cirurgiões; Doenças Cardiovasculares; Fatores de Risco; Sistemas de Saúde; Infecção/complicações; Profissionais de Saúde; Comportamento Sedentário; Epidemiologia.

Correspondência: Andre Luiz Cerqueira Almeida •

Santa Casa de Misericordia de Feira de Santana - Cardiologia - Rua Edelvira

de Oliveira, 192. CEP 44001-032, Feira de Santana, BA - Brasil

E-mail: andrealmeida@cardiol.br, andrealmeida@uefs.br

Artigo recebido em 16/11/2020, revisado em 09/05/2021,

aceito em09/06/2021

DOI: https://doi.org/10.36660/abc.20201231 e no site da Diretoria de Qualidade Assistencial da SBC, convidando os médicos especialistas em cardiologia a participarem. Adicionalmente foram enviados convites por meio de aplicativo de mensagem amplamente disponível para grupos de cardiologistas de sociedades regionais, departamentos e grupos de estudo pertencentes à SBC. Esta participação foi voluntária e secreta, não havendo a opção do cardiologista se identificar. Não houve qualquer compensação financeira ou material como retorno à participação na pesquisa. O período da coleta de dados foi de 10 de julho de 2020 a 22 de julho de 2020. O formulário online (https://wdcom.typeform.com/report/ fmQda3LQ/tOStzUhXlifR8JPj) consistiu em 28 perguntas com preenchimento obrigatório, sobre a prática assistencial e a qualidade de vida do cardiologista brasileiro durante a pandemia da COVID-19. A maioria das questões foi do tipo múltipla escolha, sendo que em muitas delas era possível responder mais de uma opção.

\section{Aspectos éticos}

Seguindo a recomendação da Resolução 510 do Conselho Nacional de Saúde, este questionário não foi encaminhado para avaliação pelo sistema CEP/CONEP, visto tratar-se de uma pesquisa de opinião pública com participantes não identificados.

\section{Análise estatística}

Foi realizada a análise descritiva dos dados obtidos na amostra. As variáveis nominais ou categóricas foram descritas por seus valores absolutos, percentagens ou proporções. As variáveis numéricas foram descritas como média e desviopadrão ou mediana e intervalo interquartil, a depender do padrão de distribuição. O teste exato de Fisher foi utilizado para testar associações entre variáveis categóricas, utilizando um nível de significância de 5\%. A análise dos dados, assim como a construção dos gráficos, foi feita com o auxílio do Excel ${ }^{\circledR}$, Microsoft $365 \AA$. As análises inferenciais foram feitas utilizando o programa estatístico Stata/SE versão 16.1, desenvolvido pela StataCorp ${ }^{\circledR}$. 


\section{Resultados}

\section{Aspectos gerais}

Um total de 1224 cardiologistas acessaram o questionário. Destes, dois recusaram a participação e 1222 responderam, representando 9,4\% dos cardiologistas adimplentes na SBC. A média de idade da população do estudo foi 47,9 \pm 11,5 anos; 711 (58,2\%) do sexo masculino. A Figura 1 mostra a distribuição dos respondentes por região do Brasil (1A), seus locais de trabalho (1B), a renda mensal antes e durante a pandemia (1C) e a reestruturação imposta à rotina de trabalho dos cardiologistas (1D).

Observou-se uma associação significativa $(p<0,001)$ entre sexo masculino e maiores faixas de renda (Tabela 1). Cardiologistas que trabalham no setor privado ou têm atividade de docência tiveram maior mudança de renda durante a pandemia $(p<0,001)$.

\section{Aspectos relacionados à renda e ao trabalho}

Houve um aumento de $37,5 \%$ no número de cardiologistas que passaram a trabalhar em três ou mais plantões por semana durante a pandemia. Por outro lado, $64 \%$ reduziram a carga horária no consultório, 22\% cancelaram aluguel de sala de consultório, $18 \%$ precisaram demitir funcionários e 9\% cancelaram investimentos em marketing (Figura 1-D).

Como reflexo da redução do retorno financeiro durante a pandemia, $15 \%$ dos cardiologistas deixaram de pagar entidades de classe. Outras medidas para redução de custos estão expressas na Figura 2A.
Quando analisamos o impacto da pandemia por faixa etária, considerando 50 anos como ponto de corte, dos resultados válidos, $56 \%$ dos entrevistados tinham menos de 50 anos e $44 \%$ tinham 50 anos ou mais. Desses dois grupos, observamos um aumento $(p<0,001)$ no número de plantões entre os médicos mais jovens, sem impactar na renda média entre os dois grupos.

Cardiologistas clínicos representaram 42\% da amostra, seguidos por ecocardiografistas (39\%), cardiopediatras (7\%), hemodinamicistas (6\%), eletrofisiologistas (4\%) e cirurgiões cardiovasculares (2\%) (Figura 3A). Dentre os ecocardiografistas, $54,5 \%$ constataram uma redução superior a $50 \%$ no volume de exames realizados/mês durante a pandemia (Figura 3B). Na hemodinâmica, $62,8 \%$ dos entrevistados relataram redução maior que $50 \%$ no volume de exames ou procedimentos no mesmo período (Figura 3-C). Entre os cirurgiões cardiovasculares, $77,3 \%$ relataram redução superior a 50\% no número de cirurgias (Figura 3-D). A subespecialidade ecocardiografia mostrou associação com redução na prática de atividade física pelos profissionais ( $p<0,001$ ).

As consultas por videoconferência no âmbito da Telemedicina foram autorizadas recentemente no Brasil. Nesta pesquisa, 30\% dos entrevistados as realizaram, porém apenas $36 \%$ foram reembolsados integralmente pelo serviço (Figura 4). Antes da pandemia, 48,8\% das mulheres ganhavam mais de $\mathrm{R} \$ 20$ mil ao mês e, durante a pandemia, houve uma redução de $63 \%$, e apenas $18 \%$ continuaram com essa renda. Entre os homens a redução foi de $45 \%$ (81,2\% para 44,6\%) (Figura 5). Apenas 7,6\% das mulheres e $1,8 \%$ dos homens ganhavam menos de R\$10mil por mês antes da pandemia, e esse número

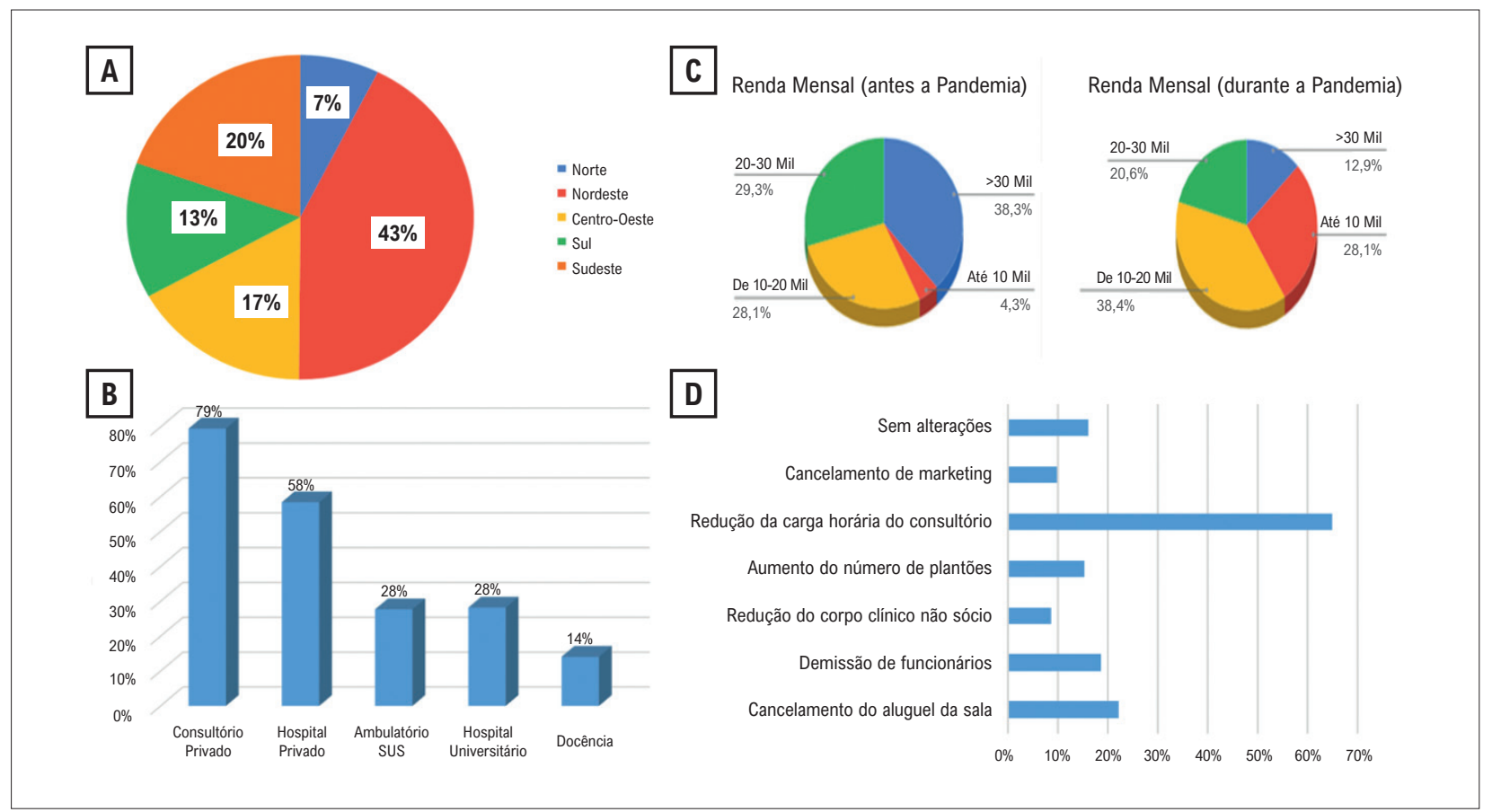

Figura 1 - Distribuição dos cardiologistas participantes do estudo por região geográfica (A), local de trabalho (B); renda mensal antes e durante a pandemia (C), e reestruturação no trabalho devido à pandemia (D). 


\section{Carta Científica}

Tabela 1 - Associação entre as variáveis avaliadas na pesquisa sobre o impacto da pandemia da COVID-19 na vida de cardiologistas brasileiros $(n=1222)$

\begin{tabular}{lcc}
\hline Variável $\mathbf{0 1}$ & Redução de atividade sexual & Associação (valor de p) \\
\hline Sexo feminino & Maiores faixas de renda & $<0,001$ \\
\hline Sexo masculino & Maior mudança de renda & $<0,001$ \\
\hline Trabalho no setor privado & Maior mudança de renda & $<0,001$ \\
\hline Atividade de docência & Aumento de plantões & $<0,001$ \\
\hline Idade $<50$ anos & Redução na atividade física & $<0,001$ \\
\hline Subespecialidade ecocardiografia & Redução na atividade física & $>0,05$ \\
\hline Renda mensal & Redução na atividade física & $>0,05$ \\
\hline Ganho de peso & Redução na atividade física & $>0,05$ \\
\hline Sexo & Mudanças na rotina do trabalho & $>0,05$ \\
\hline Sexo & Medidas adotadas para reduzir os custos & $>0,05$ \\
\hline Faixa etária & & \\
\hline
\end{tabular}

passou para $38,2 \%$ e $20,8 \%$, respectivamente, durante a pandemia (Figura 5).

As medidas adotadas para reduzir os custos durante a pandemia não tiveram associação significativa com a faixa etária da amostra (Figura 6).

\section{Aspectos relacionados às mudanças de rotina e de estilo de vida}

Dos entrevistados, $69 \%$ praticavam atividade física antes da pandemia. Desses, 63\% reduziram ou suspenderam a prática da atividade física durante a pandemia. Doze por cento experimentaram conflito familiar (quatro relatos de violência doméstica); $17 \%$ passaram a fazer uso de antidepressivos ou ansiolíticos e 11\% aumentaram o uso de drogas lícitas (Figura 2B). Não houve associação de redução da prática de atividade física com o sexo ou com a renda ( $p>0,05)$.

Considerando as últimas quatro semanas da pandemia, $44 \%$ dos entrevistados relataram ganho de peso, sendo que $13 \%$ relataram ganho superior a $3 \mathrm{~kg}$. Em 35\% dos casos, o peso manteve-se estável. Neste mesmo período de observação, $26 \%$ relataram aumento do consumo de bebidas alcoólicas, enquanto $30 \%$ referiram que o consumo permaneceu estável. Não houve associação entre ganho de peso e mudança na atividade física ( $p>0,05)$.

Dos entrevistados, 40,2\% relataram diminuição na frequência das relações sexuais, para 41,6\% essa frequência manteve-se estável, e apenas 7,4\% relataram aumento na frequência de relações (Figura 7). Essa redução foi mais significativa nos profissionais do sexo feminino $(p<0,001)$.

\section{Aspectos relacionados à infecção pela COVID-19}

Do total de investigados, 54,9\% mostraram moderada ou muita preocupação em trabalhar na linha de frente do combate à COVID-19. Até o final do período pesquisado (22/07/2020), 20\% dos cardiologistas entrevistados tinham tido infecção sintomática confirmada pelo novo coronavírus. Em 1,8\% dos casos, os sintomas foram graves, necessitando internamento, ao passo que os sintomas foram leves e sem necessidade de hospitalização em 15\% dos que responderam ao questionário. Em 3\% dos casos a infecção foi confirmada, mas a evolução foi assintomática.

\section{Discussão}

O presente estudo relata os resultados da primeira pesquisa nacional que avaliou o impacto causado pela pandemia da COVID-19 nas questões profissionais, financeiras, de saúde (física e mental), e de estilo de vida dos médicos cardiologistas brasileiros. As respostas de 1222 cardiologistas, distribuídos por todas as regiões do Brasil, demonstraram um forte impacto em todas as áreas investigadas. Foi nítida a redução nos ganhos financeiros, associada à redução da carga horária no consultório e à necessidade de aumento no número de plantões semanais. Como consequência, a quitação de alguns compromissos financeiros ficou comprometida, incluindo pagamento de entidade de classe, cursos de aprimoramento profissional e custeio da educação dos filhos. Notamos ainda uma importante redução na prática de atividades físicas e de relações sexuais durante a pandemia, além de aumento nos conflitos familiares e no uso de antidepressivos e ansiolíticos. Quase metade dos cardiologistas relataram aumento no peso corporal e $25 \%$ relataram aumento na ingestão de bebidas alcoólicas.

Assim como aconteceu com os cardiologistas brasileiros, uma pesquisa divulgada pela British Medical Association em julho de 2020 apontou que 39,5\% dos médicos britânicos relataram redução nos ganhos financeiros e 30,7\% referiram condições de saúde mental relacionadas ou agravadas pelo seu trabalho durante a pandemia da COVID-19, como depressão, ansiedade, estresse, esgotamento e sofrimento emocional. ${ }^{4} \mathrm{Em}$ recente pesquisa realizada com 766 urologistas brasileiros, $54,8 \%$ relataram redução nos ganhos financeiros superior a $50 \%$ durante a pandemia da COVID-19, 32,9\% relataram ganho de peso, 60,0\% redução na prática de atividade física, $39,9 \%$ aumentaram o consumo de álcool e 34,9\% referiram redução na atividade sexual. ${ }^{5}$

Vários níveis de evidências sugerem que a inatividade física pode provocar importantes repercussões na fisiologia 

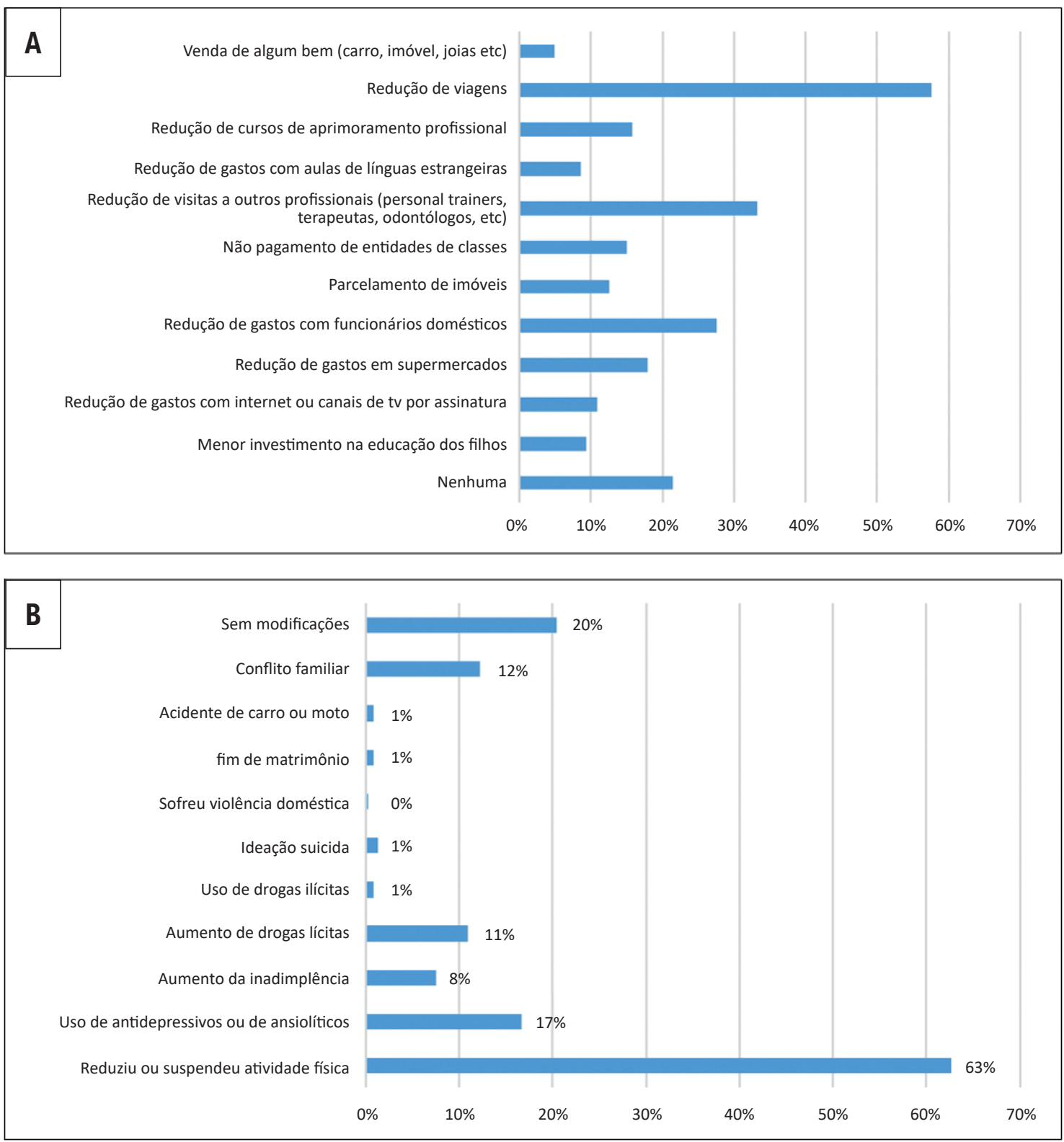

Figura 2 - Medidas de redução de custos (A) mudanças no estilo de vida (B) durante a pandemia da COVID-19 relatadas por cardiologistas brasileiros.

cardiovascular. ${ }^{6}$ As atividades físicas dos cardiologistas estudados foram reduzidas ou suspensas em $63 \%$ dos casos, o que pode ter impactado nos $44 \%$ que tiveram aumento de peso superior a $3 \mathrm{~kg}$. Uma publicação recente relacionou a redução de atividade física e ganho de peso com o aumento do risco de doenças cardiovasculares, além de alertar sobre outros perigos da obesidade. ${ }^{7}$

Pelo nosso levantamento, 26\% passaram a ingerir mais bebidas alcoólicas, enquanto $40 \%$ dos entrevistados afirmaram uma diminuição no número de relações sexuais em comparação a antes da pandemia. É plausível a associação do aumento do consumo de álcool e da exacerbação de conflitos familiares com o impacto psicológico causado pelo isolamento social prolongado. O aumento de conflitos familiares foi relatado por $12 \%$ dos nossos entrevistados, incluindo quatro profissionais que sofreram violência doméstica. Esse número pode ser bem maior, posto que houve aumento desses casos durante o período de isolamento social em outros países como a China (onde os casos triplicaram), ${ }_{1}^{8}$ Reino Unido, Estados Unidos e França (atingindo 36\%). ${ }^{9}$ No Brasil, esse índice chegou a aumentar em 17\%, conforme dados do Ministério da Mulher. ${ }^{10}$

O governo brasileiro regularizou e autorizou temporariamente o atendimento remoto de pacientes por 


\section{Carta Científica}

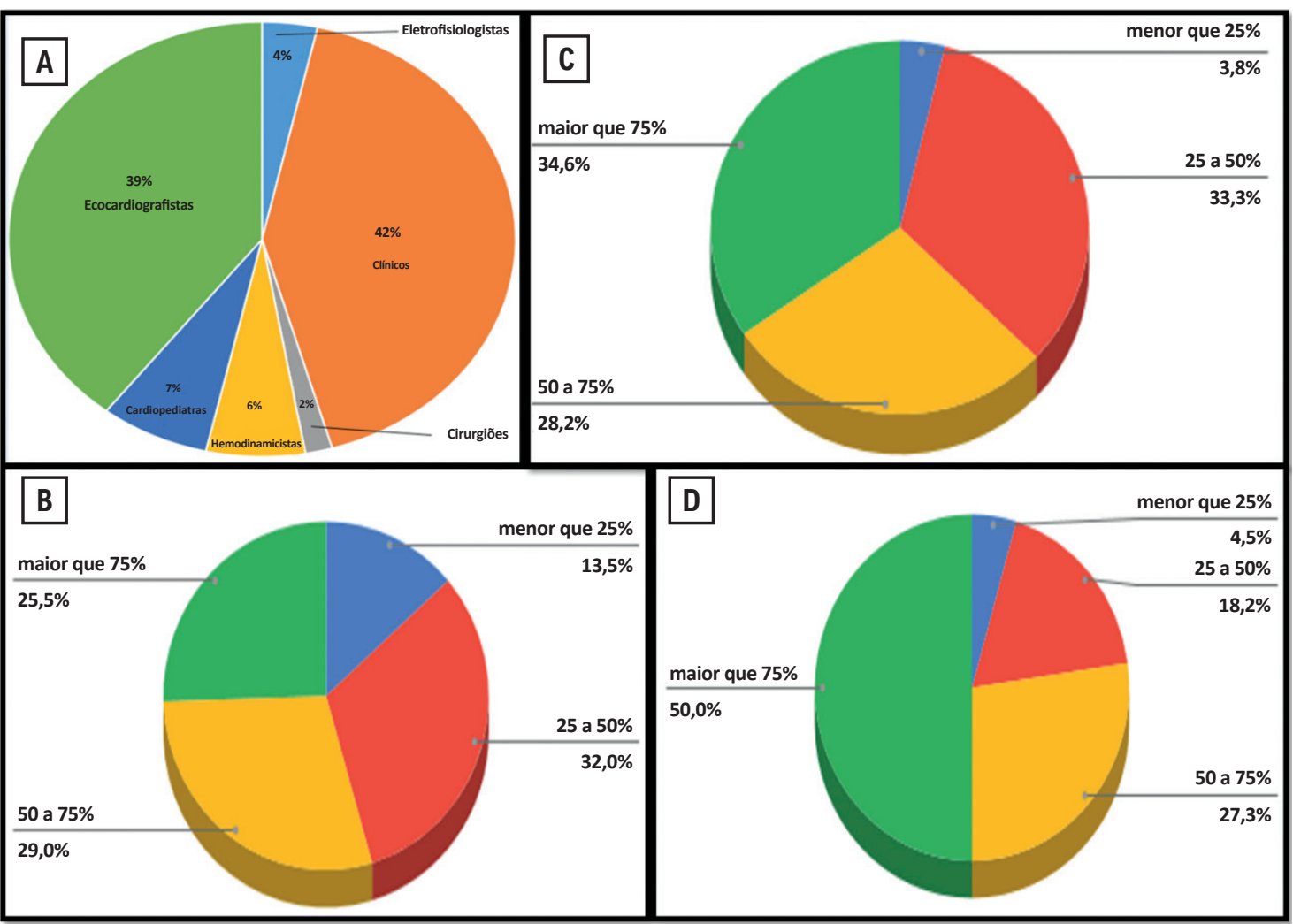

Figura 3 - Distribuição dos cardiologistas participantes ( $n=1222)$ por subespecialidade $(A)$; e por porcentagem de profissionais que relataram redução nos procedimentos de ecocardiografia (B); hemodinâmica (C) e cirurgia cardiaca (D)

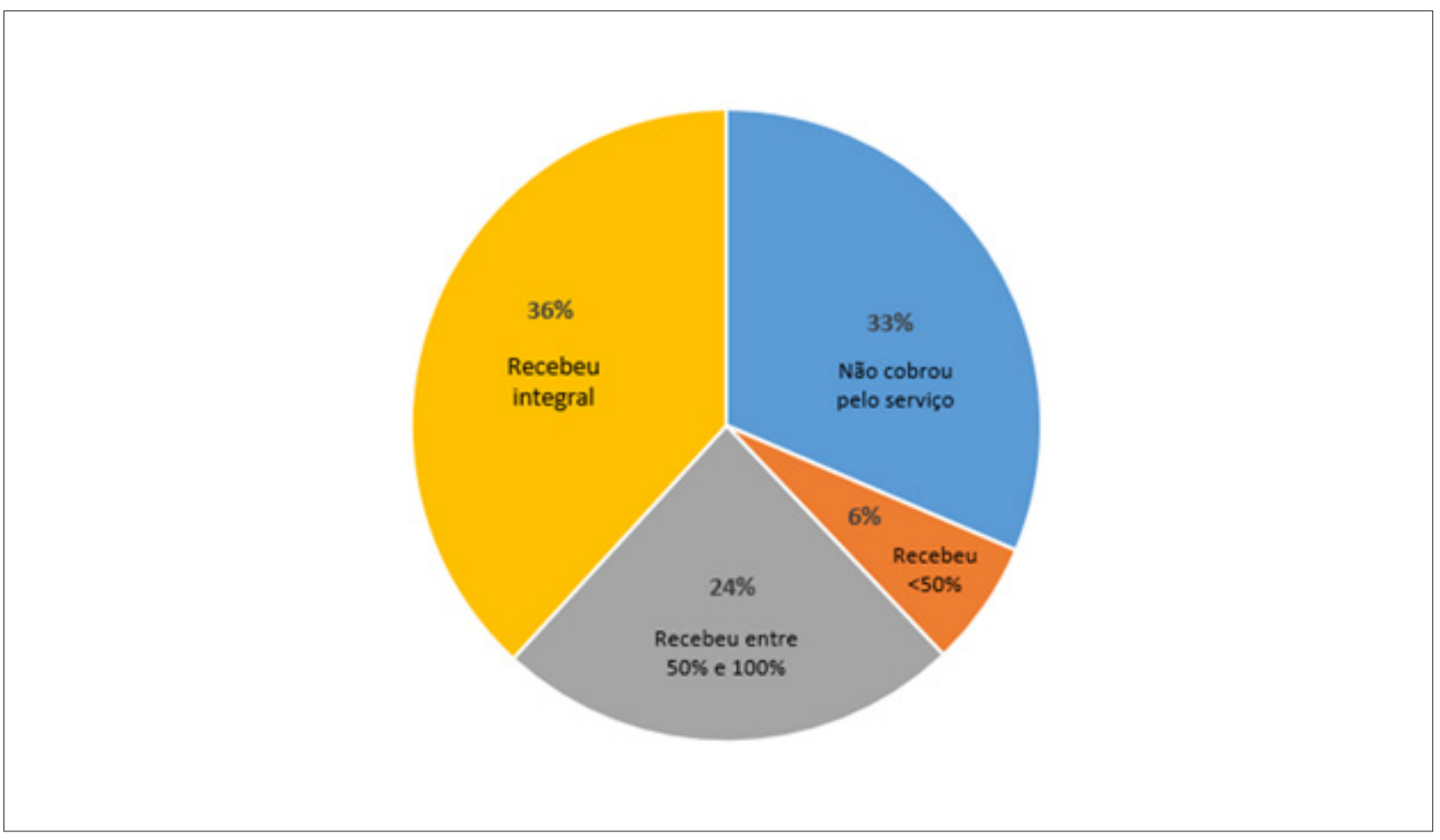

Figura 4 - Reembolso das consultas por videoconferência realizadas durante a pandemia da COVID-19 por cardiologistas brasileiros ( $n=1222)$. 


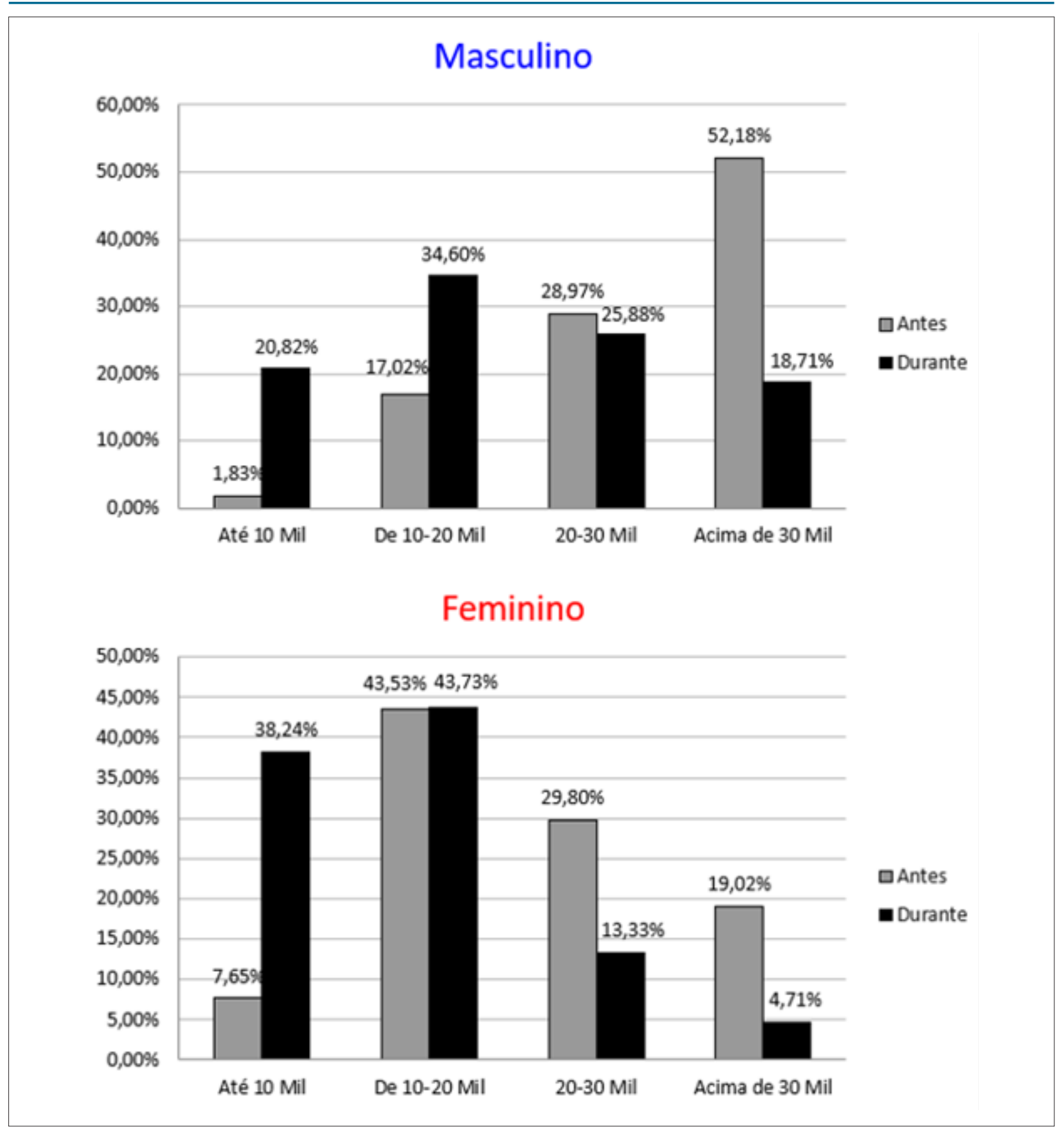

Figura 5 - Distribuição dos cardiologistas brasileiros (n=1222) quanto à renda e sexo antes e durante a pandemia da COVID-19.

meio da telemedicina no Brasil. ${ }^{11}$ Mesmo em fase ainda inicial, precipitada pelo isolamento social imposto pela pandemia, $30 \%$ dos cardiologistas que responderam ao questionário afirmaram ter realizado teleconsultas, embora apenas 36\% destes tenham sido reembolsados integralmente pelo serviço. Como comparação, 38,7\% dos urologistas brasileiros relataram ter realizado teleconsultas, com mais de 50\% informando reembolso pelo serviço prestado. ${ }^{5}$

Em 2017, um questionário foi enviado via e-mail a todos os 13462 cardiologistas adimplentes associados à SBC; 2101
(15,6\%) responderam efetivamente, sendo 1509 (71,8\%) homens e 592 (28,2\%) mulheres. ${ }^{12}$ Dos 1222 (9,1\% dos sócios da SBC) que responderam ao nosso questionário, 711 (58,2\%) eram homens. A faixa etária foi semelhante aos respondedores das duas enquetes, sendo que 51,3\% dos que responderam à pesquisa da SBC tinham mais de 50 anos, contra 44\% dos respondedores do nosso trabalho. Em relação à distribuição geográfica, 54\% dos sócios adimplentes da SBC estão na região Sudeste, $19 \%$ no Nordeste, $15 \%$ no Sul, $8 \%$ na região Centro-Oeste e $3 \%$ no Norte. Dentre os que responderam 


\section{Carta Científica}

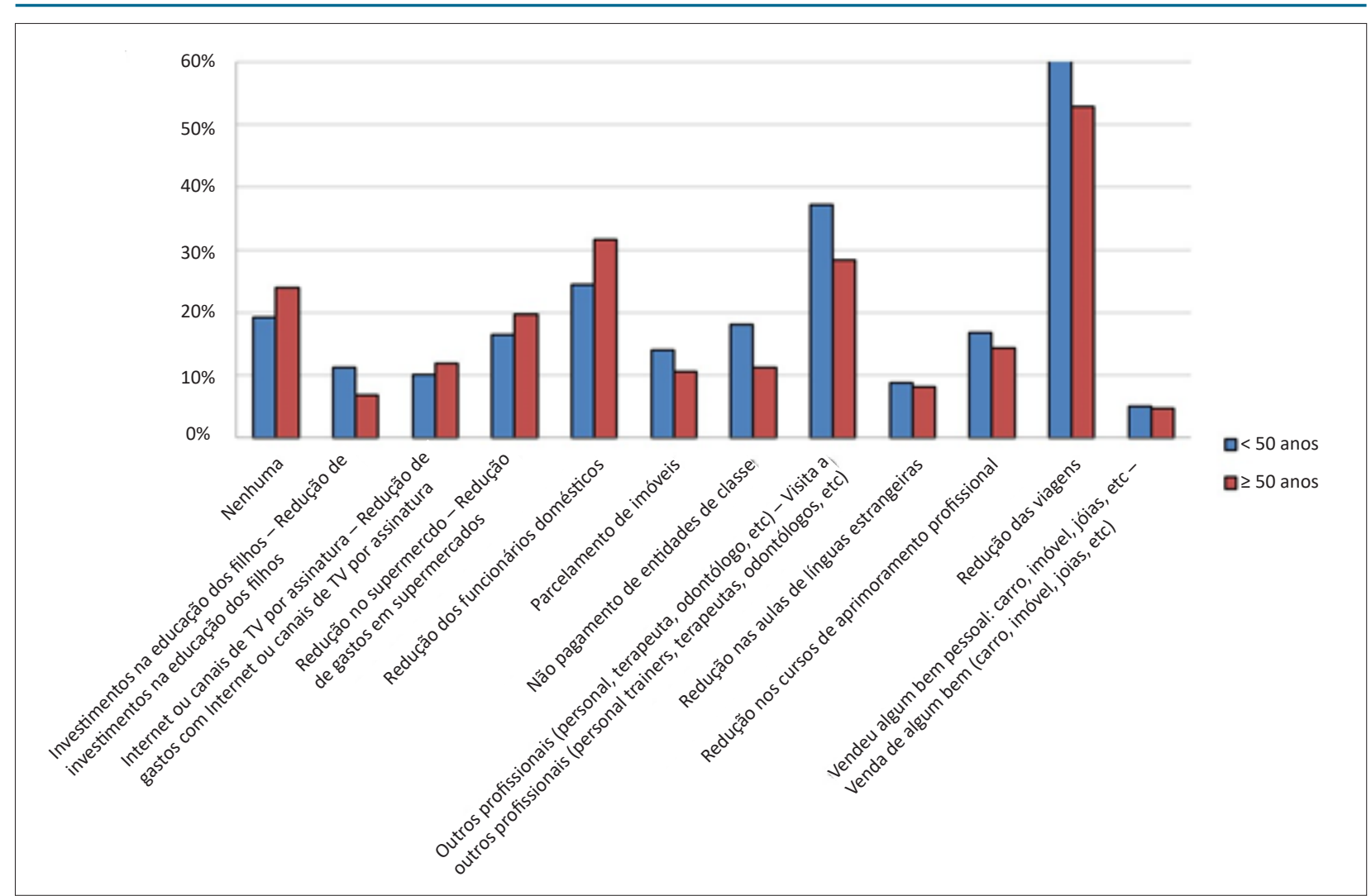

Figura 6 - Relação da faixa etária com as medidas para redução de custos durante a pandemia.

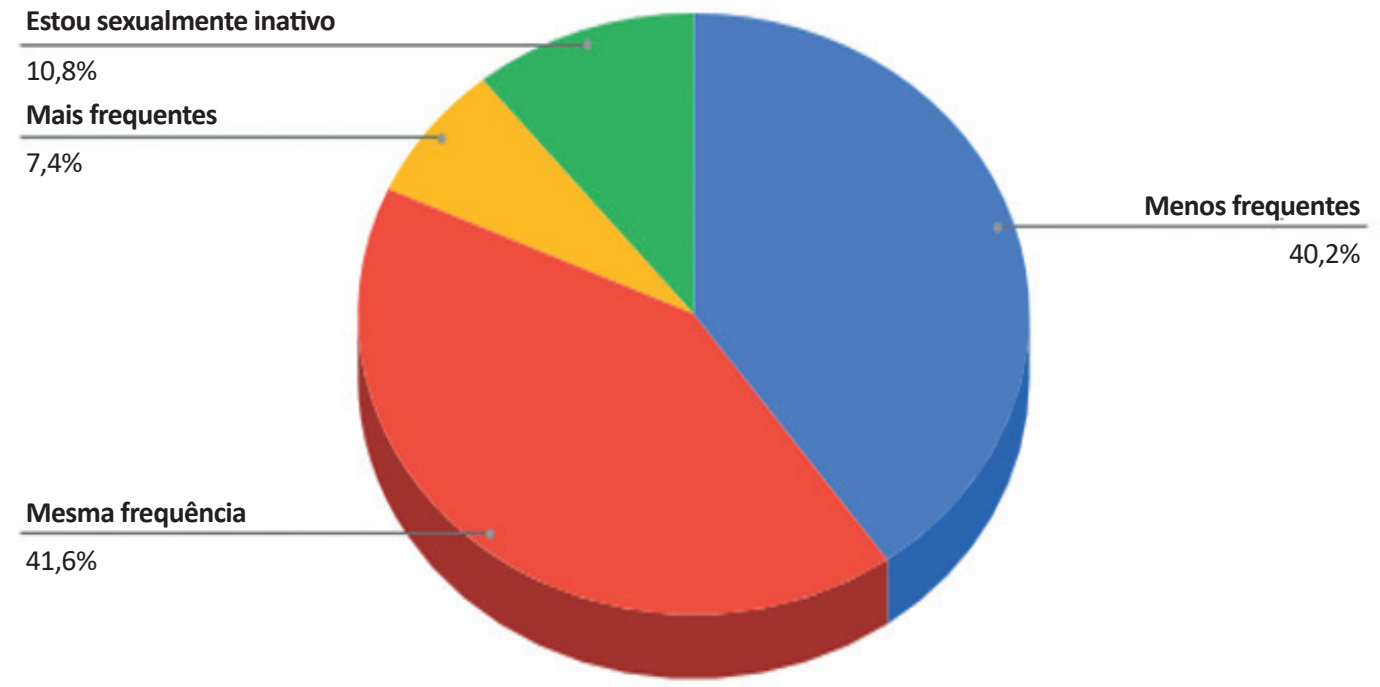

Figura 7 - Frequência de relações sexuais relatadas pelos cardiologistas durante a pandemia $(n=1222)$ 
ao nosso questionário, $43 \%$ estavam na região Nordeste, $17 \%$ no Centro-Oeste, $13 \%$ no Sul, $20 \%$ no Sudeste e $7 \%$ no Norte do Brasil.

O presente estudo possui algumas limitações inerentes aos estudos transversais baseados em resposta a um questionário. O número de respondentes da atual pesquisa representa pouco menos de $10 \%$ do número de cardiologistas associados à SBC. A distribuição geográfica dos participantes da pesquisa é diferente da dos sócios da SBC. Outro ponto relevante é a impossibilidade de comprovar as respostas ou esclarecê-las; porém, apesar da incerteza da veracidade das respostas, o estudo foi coerente com outros dados publicados em âmbito nacional e internacional. Embora nosso estudo tenha encontrado algumas associações interessantes e com significância estatística, tais achados devem ser considerados meramente exploratórios, não podendo desconsiderar a possibilidade de achados falso-positivos pela quantidade de testes de hipóteses realizados.

\section{Conclusão}

Esse estudo demonstra o impacto negativo da pandemia de COVID-19 no trabalho, renda, saúde e estilo de vida dos médicos cardiologistas brasileiros. São dados de extrema relevância que ajudarão no planejamento em futuros cenários de caos como o atual enfrentamento pandêmico.

\section{Referências}

1. Almeida ALC, Santo TME, Mello MSS, Cedro AV, Lopes NL, Ribeiro APMR, et al. Repercussions of the COVID-19 Pandemic on the Care Practices of a Tertiary Hospital. Arq Bras Cardiol. 2020; 115(5):862-70. doi: 10.36660/ abc. 20200436 .

2. Adam S, Zahra SA, Chor CYT, Khare Y, Harky A. COVID-19 pandemic and its impact on service provision: A cardiology prospect. Acta Cardiol. 2020 Jul 10:1-8. doi: 10.1080/00015385.2020.1787636.

3. Collins GB, Jenner WJ, Kaier TE, Bhattacharyya S. COVID-19: A United Kingdom National Health Service Cardiology Perspective. JACC Case Rep. 2020;2(9):1426-8. doi: 10.1016/j.jaccas.2020.04.024.

4. COVID-19: analysing the impact of coronavirus on doctors. 2020. \{Internet]. [Cited in 2020 July 13] Available from: <https://www.bma.org.uk/adviceand-support/covid-19/what-the-bma-is-doing/covid-19-analysing-theimpact-of-coronavirus-on-doctors $>$.

5. Gomes CM, Favorito LA, Henriques JVT, Canalini AF, Anzolch KMJ, de Carvalho Fernandes R, et al. Impact of COVID-19 on clinical practice, income, health and lifestyle behavior of Brazilian urologists. Int Braz J Urol. 2020;46(6):1042-71. doi: 10.1590/S1677-5538.IBJU.2020.99.15.

6. Schmid D, Ricci C, Baumeister SE, Leitzmann MF. Replacing Sedentary Time with Physical Activity in Relation to Mortality. Med Sci Sports Exerc. 2016;48(7):1312-9. doi:10.1249/MSS.0000000000000913.

\section{Contribuição dos autores}

Concepção e desenho da pesquisa: Almeida ALC, Melo M, Barberato SH; Obtenção de dados: Almeida ALC, Melo M, Rodrigues REF, Almeida PAA, Barberato SH; Análise e interpretação dos dados, Redação do manuscrito e Revisão crítica do manuscrito quanto ao conteúdo intelectual importante: Almeida ALC, Melo M, Rodrigues REF, Botelho LF, Almeida PAA, Barberato SH; Análise estatística: Botelho LF.

\section{Potencial conflito de interesse}

Não há conflito com o presente artigo

\section{Fontes de financiamento}

O presente estudo não teve fontes de financiamento externas.

\section{Vinculação acadêmica}

Não há vinculação deste estudo a programas de pósgraduação.

\section{Aprovação ética e consentimento informado}

Este artigo não contém estudos com humanos ou animais realizados por nenhum dos autores.

7. Clemmensen C, Petersen MB, Sorensen TIA. Will the COVID-19 pandemic worsen the obesity epidemic? Nat Rev Endocrinol. 2020;16(9):469-70. doi: 10.1038/s41574-020-o387-z

8. China's Hidden Epidemic: Domestic Violence ,2020 [Internet] [Cited in 2020 Aug 18] Available from: https://thediplomat.com/2020/04/chinashidden-epidemic-domestic-violence

9. Network E. Domestic violence increases in France during COVID-19 lockdown [Internet]. [Cited in 2020 Aug 18] Available from: https://www. euractiv.com/section/politics/news/domestic-violence-increases-in-franceduring-covid-19-lockdown/

10. Brasil. Ministério da Saúde. Ministério da Mulher, da Família e dos Direitos Humanos [Internet] [Cited in 2020 Aug 12] Disponível em: https://www. gov.br/mdh/pt-br/navegue-por-temas/politicas-para-mulheres/ligue-180

11. Diário Oficial da União - Portaria no 467, de 20 de março de 2020. Available from: <http://www.in.gov.br/en/web/dou/-/portaria-n-467-de-20-demarcode-2020-249312996>; <Acessado em August 29, 2020>.

12. Faganello LS, Pimentel M, Polanczyk CA, Zimerman T, Malachias MVB, Dultra OP, et al. O perfil do cardiologista brasileiro - uma amostra de sócios da Sociedade Brasileira de Cardiologia. Arq Bras Cardiol. 2019; 113(1):62-8. doui: 10.5835/abc.20190089 